University of Wollongong

Research Online

Australian Institute for Innovative Materials -

Papers

Australian Institute for Innovative Materials

$1-1-2015$

Super-paramagnetic particles chemically bound to luminescent diamond: single nano-crystals probed with optically detected magnetic resonance

Taras Plakhotnik

University of Queensland

Haroon Aman

University of Queensland

Shaohua Zhang

University of Wollongong, sz826@uowmail.edu.au

Zhen Li

University of Wollongong, zhenl@uow.edu.au

Follow this and additional works at: https://ro.uow.edu.au/aiimpapers

Part of the Engineering Commons, and the Physical Sciences and Mathematics Commons

Research Online is the open access institutional repository for the University of Wollongong. For further information contact the UOW Library: research-pubs@uow.edu.au 


\title{
Super-paramagnetic particles chemically bound to luminescent diamond: single nano-crystals probed with optically detected magnetic resonance
}

\begin{abstract}
We have synthesized novel composite particles made of single-domain nanomagnets chemically bound to nanocrystals of diamond. Optically detected magnetic resonance spectra of nitrogen-vacancy centers in diamond allowed us to estimate the magnetic field of the nanomagnets and to observe the saturation of their magnetization when an external field of a few tens of $\mathrm{mT}$ has been applied. The saturation effect is in agreement with the size of the domains measured using transmission electron microscopy and a simple model of magnetization.
\end{abstract}

\section{Keywords}

magnetic, resonance, super, paramagnetic, particles, chemically, bound, luminescent, diamond, single, nano, crystals, probed, optically, detected

\section{Disciplines}

Engineering | Physical Sciences and Mathematics

\section{Publication Details}

Plakhotnik, T., Aman, H., Zhang, S. \& Li, Z. (2015). Super-paramagnetic particles chemically bound to luminescent diamond: single nano-crystals probed with optically detected magnetic resonance. The Journal of Physical Chemistry C: Energy Conversion and Storage, Optical and Electronic Devices, Interfaces, Nanomaterials, and Hard Matter, 119 (34), 20119-20124. 


\section{Super-Paramagnetic Particles Chemically Bound}

\section{to Luminescent Diamond: Single Nano-Crystals Probed with Optically Detected Magnetic}

\section{Resonance}

Taras Plakhotnik, ${ }^{*}{ }^{\dagger}$ Haroon Aman, ${ }^{\dagger}$ Shaohua Zhang, ${ }^{\ddagger}$ and Zhen $\mathrm{Li}^{\ddagger}$

School of Mathematics and Physics, The University of Queensland, St Lucia, QLD 4072, Australia, and Institute for Superconducting and Electronic Materials, Australian Institute of Innovative Materials, University of Wollongong, Squires Way, North Wollongong, NSW 2500, Australia

E-mail: taras@physics.uq.edu.au

\footnotetext{
${ }^{*}$ To whom correspondence should be addressed

† School of Mathematics and Physics, The University of Queensland, St Lucia, QLD 4072, Australia

${ }^{\ddagger}$ Institute for Superconducting and Electronic Materials, Australian Institute of Innovative Materials, University of Wollongong, Squires Way, North Wollongong, NSW 2500, Australia
} 


\begin{abstract}
We have synthesised novel composite particles made of single-domain nano-magnets chemically bound to nano-crystals of diamond. Optically detected magnetic resonance spectra of nitrogen-vacancy centers in diamond allowed to estimate the magnetic field of the nano-magnets and to observe the saturation of their magnetisation when an external field of few tens of $\mathrm{mT}$ has been applied. The saturation effect is in agreement with the size of the domains measured using transmission electron microscopy and a simple model of magnetisation.
\end{abstract}

\title{
Introduction
}

Single-domain magnetic particles (also called super-paramagnetic nanoparticles) are small crystals of ferromagnetic material where all the unpaired electronic spins are aligned parallel to each other. ${ }^{1}$ Properties of such particles are interesting for a number of reasons. For example, they define the smallest size of a recording magnetic material which can reliably store information. ${ }^{2}$ The nano-magnets also have found applications in biotechnology, for magnetic separation and isolation of specific molecules or other particles bound to the nano-magnets. ${ }^{3}$ Magnetic nano-particles are used in medical diagnostics, magnetic resonance imaging and hypothermal therapy. ${ }^{4}$ Another interesting direction for applications is computing based on nano-magnetic logic. ${ }^{5}$ Such a computer potentially could use orders of magnitude less energy than modern highly integrated electronic circuits.

Properties of single-domain magnets such as, for example, the rate of spontaneous magnetisation reversal ${ }^{6-8}$ and the transition from a single-domain superparamagnetic particle to ferromagnetic are strongly dependent on their size. Therefore even a relatively narrow distribution of particle dimensions affects the ensemble measurements. ${ }^{7}$ Observation of a single particle eliminates the effect of the size inhomogeneity and isolated single-domain magnets have been observed using, for example, micro-SQUID magnetometry to study magnetisation reversal ${ }^{9}$ which is still a hot topic of research. ${ }^{10}$ 
Nitrogen-vacancy centers, in diamond were identified about 40 years ago. ${ }^{11,12}$ They possess a set of unique properties enabling applications in quantum information processing, ${ }^{13,14}$ luminescent labeling, ${ }^{15}$ sub-diffraction imaging ${ }^{16}$ and as sensitive and optically addressable nano-sensors of electromagnetic fields ${ }^{17}$ and temperatures. ${ }^{18,19}$ While full scale quantum computing is a remote future, the nano-sensing field is at the stage of practical realization. For example, noise floor of $3.5 \mathrm{~K} \mathrm{~s}^{1 / 2}$ has been achieved in all-optical measurements of the temperature using a method which is compatible with standard optical microscopes used in biological research. ${ }^{20}$ More recently ${ }^{21}$ the results have been improved by an order of magnitude to reach $0.3 \mathrm{~K} \mathrm{~s}^{1 / 2}$.

The NV centers have been shown to be among the most sensitive detectors of magnetic fields. The electronic ground state of NV-centers has spin 1 and the magnetic field dependent splitting between its $m= \pm 1$ and $m=0$ states can be measured with very high sensitivity using optically detected magnetic resonance (ODMR) technique which exploits the dependence of the luminescence intensity of NV centers on the magnetic quantum number $\mathrm{m}$. The states with $m= \pm 1$ can decay non radiatively from the electronically excited state ${ }^{3} E$ first to intermediate singlet states and then to the $\mathrm{m}=0$ level of ${ }^{3} \mathrm{~A}$, the electronic ground state. ${ }^{22}$ The $m=0$ state of ${ }^{3} E$ decay only directly to $m=0$ level of the ${ }^{3} A$ state. Under continuos optical pumping, such selectivity populates the $m=0$ spin level. Applied in resonance to the $\Delta \mathrm{m}= \pm 1$ transitions, Radio Frequency (RF) magnetic field repopulates the $\mathrm{m}= \pm 1$ levels and causes the luminescence intensity to decrease (population of $m= \pm 1$ reopens the not radiative decay channel).

The theoretical noise floor of NV-magnetometry (the minimum detectable change of the field multiplied by the square root of the measurement time) can be ${ }^{23}$ as low as few fT $\mathrm{s}^{1 / 2}$. The experimentally demonstrated noise floor is about $3 \mathrm{nT} \mathrm{s} \mathrm{s}^{1 / 2}$ with NV -centers in bulk diamond, ${ }^{24}$ about $100 \mathrm{nT} \mathrm{s}{ }^{1 / 2}$ with the centre being close to the surface, ${ }^{25}$ and about $500 \mathrm{nT}$ $\mathrm{s}^{1 / 2}$ in a nanocrystal. ${ }^{24}$ The magnetic sensitivity of NV- centers enables detection of 19-nm magnetic particles using bulk diamond crystals. ${ }^{26}$ Because a single crystal of diamond can 
host NV-centers with 4 different orientations of their axes, it is possible to perform a vector magnetometry, ${ }^{27,28}$ that is to determine the magnitude and the direction (relative to the crystal axes) of an unknown magnetic field vector.

Here we present the first step towards development of composite nanoparticles where a single nano-diamond is connected to a single nano-magnet. Such particles represent a new class of nanosystems with unique optical and magnetic properties. For example, the NVcenters in diamond may work as sensors of the temperature, which is a critical parameter for the hypothermal treatment. $\mathrm{Fe}_{3} \mathrm{O}_{4}$ nanoparticles have been demonstrated recently to exhibit high heating efficiency but a wide size distribution. ${ }^{29}$ The coupled nano-diamonds could measure each particle temperature individually and investigate the effect of the sizeinhomogeneity on the heating. On the other hand, the temperature sensitivity of the proposed composite particles can be higher than the sensitivity of plain NV-centers due to the temperature dependent magnetisation of the nano-magnets. ${ }^{30}$ The use of the composite particle would reduce the size of such a sensor dramatically (to tens of nanometers) in comparison to the apparatus used in the original publication. ${ }^{30}$ Magnetic sensitivity of NV-centers can also be used to read the state of a nano magnetic register or to probe magnetisation reversal on a single particle level without the limitations of the SQUID or scanning-probe microscopy. In this paper, we aim at synthesis of novel composite particles made of a single nano-diamond of approximately 40-nm across chemically attached to single-domain nano-magnets of about $12 \mathrm{~nm}$ in diameter. In addition to the synthesis, the goal of this work is to demonstrate that NV-centers in nano-diamonds can be used to detect the field created by the nanomagnets and thus to confirm their presence on the surface of the diamonds. 


\section{Experimental}

\section{Materials}

Iron (III) chloride hexahydrate $\left(\mathrm{FeCl}_{3} \cdot 6 \mathrm{H}_{2} \mathrm{O}, 97 \%\right)$, iron (II) sulfate heptahydrate $\left(\mathrm{FeSO}_{4}\right.$. $\left.7 \mathrm{H}_{2} \mathrm{O}, \geq 99 \%\right)$, ammonium hydroxide solution $\left(\mathrm{NH}_{3} \cdot \mathrm{H}_{2} \mathrm{O}, 28 \%\right)$, hydrochloric acid $(\mathrm{HCl}$, 37\%), (3-aminopropyl)triethoxysilane (APS, 99\%), ammonium hydroxide solution (28.0$30.0 \% \mathrm{NH}_{3}$ basis) were purchased from Sigma-Aldrich.

\section{Experimental Methods}

The $\mathrm{Fe}_{3} \mathrm{O}_{4}$ magnetic nanoparticles were prepared by a coprecipitation method. Typically, $50 \mathrm{~mL}$ Mili-Q water was purged with nitrogen to remove oxygen and heated to $100{ }^{\circ} \mathrm{C}$ in an oil bath $\left(130{ }^{\circ} \mathrm{C}\right)$ to reflux. Meanwhile, $\mathrm{FeCl}_{3} \cdot 6 \mathrm{H}_{2} \mathrm{O}(0.1378 \mathrm{~g}, 0.51 \mathrm{mmol})$ and $\mathrm{FeSO}_{4} \cdot 7 \mathrm{H}_{2} \mathrm{O}$ $(0.0701 \mathrm{~g}, 0.252 \mathrm{mmol})$ were dissolved in concentrated $\mathrm{HCl}(1 \mathrm{~mL})$. Then, the mixture of iron precursors was quickly injected into the hot water, followed by the addition of concentrated ammonia solution (15 mL, 28\%). The solution became black immediately, and the resultant black solution was refluxed for $2 \mathrm{~h}$. The as-obtained black precipitates were washed thoroughly with deionized water and absolute alcohol several times, respectively, and finally re-dispersed in Mili-Q water for further use.

The batch of nano-magnets has been characterised using High Resolution Transmission Electron Microscopy (HRTEM), X-ray Diffraction (XRD) and Selected Area Electron Diffraction ( SAED). The data (presented in the Supporting Information) confirm the synthesis of super-paramagnetic nano particles.

The diamond particles were functionalised with APS. Briefly, $10 \mu \mathrm{L}$ APS was added to $10 \mathrm{~mL}$ of an ethanol dispersion of diamond containing $0.68 \mathrm{ml} \mathrm{NH}_{4} \mathrm{OH}$ and $1 \mathrm{~mL} \mathrm{H}_{2} \mathrm{O}$. This mixture was stirred for $12 \mathrm{~h}$ at room temperature. The resultant product was purified via centrifugation (7830 rpm) with ethanol and water several times and re-dispersed in $1 \mathrm{~mL}$ $\mathrm{H}_{2} \mathrm{O}$ for further use. 
Electrostatic self-assembly approach was explored to prepare $\mathrm{C} / \mathrm{Fe}_{3} \mathrm{O}_{4}$. Briefly, $20 \mu \mathrm{L}$ as-prepared Magnetic Nanoparticles solution was slowly added to $1 \mathrm{~mL}$ as-made AminoFunctionalize Diamond Particles dispersion with room temperature stirring. Six hours later, the mixture was separated by centrifugation and washed with water for further use.

Diamond crystals with a mean size of about $40 \mathrm{~nm}$ prepared from synthetic sub-micron diamond powder by irradiation with 40-keV a-particles followed by annealing at about 1070 $\mathrm{K}$ to increase concentration of NV-centers and milled down to the size of nano-particles. The estimated concentration of NV centers in the diamond nanocrystals was about $12 \mathrm{ppm}$ from ensemble characteristcs. ${ }^{15}$ The water suspension of the nano-diamonds covered with single-domain magnets was casted on a spinning surface of a fused quartz substrate. The substrate was then placed in the focal plane of a microscope objective (Olympus, NA 0.9) and illuminated with $532 \mathrm{~nm}$ wavelength laser light. The laser illuminated spot on the surface of the slide had approximately a two-dimensional Gaussian shape and 30 microns diameter. Near infrared emission of $\mathrm{NV}^{-}$centers in diamond was collected with the same microscope objective and imaged on the photo sensitive matrix of a CCD (Andor iXon). The spectra were obtained with 532-nm excitation wavelength. A long-pass filter (Semrock) transmitting only light at longer than 600-nm wavelength was placed between the objective and the CCD. The external magnetic field was created by a cylindrical permanent magnet of $4 \mathrm{~mm}$ in diameter and $6 \mathrm{~mm}$ long. The strength of the field could be regulated between 0 and $0.4 \mathrm{~T}$ by the distance between the magnet and the sample. The RF field was produced with a U-shaped golden wire deposited on the surface of the substrate.

Transmission electron microscope (TEM) images have been obtained with JEOL 2011, $200 \mathrm{keV}$ electron microscope. 


\section{Results and Discussion}

A TEM image of the sample (Fig. 1) shows a pile of nano-magnets and nano-diamonds. The magnets are seen as approximately round dark objects. The mean and the standard deviation of their diameters have been estimated from the image to be about $12 \mathrm{~nm}$ and 2 $\mathrm{nm}$ respectively. The nano-diamonds are a bit more transparent, larger and have irregular shapes. The dimensions of the nano diamonds are widely distributed between 10 and 70 $\mathrm{nm}$. The concentration of the nano-magnets indicates that many magnets can be attached to a single diamond crystal but the coverage of the diamond surface is quite inhomogeneous. There are many factors which could cause the difference in the coverage. The main reason is that defects on the surface of nanodiamonds which actually serve as active sites for subsequent coating with (3-aminopropyl)triethoxysilane (APS) are usually dependent on particle size and their distribution on the surface is very inhomogeneous even for the same batch of nano-diamonds. Another reason is that these nano-diamonds are only dispersed in water rather than dissolved and they couldn't form homogenous solution during coating which also leads to differences in the coating density.

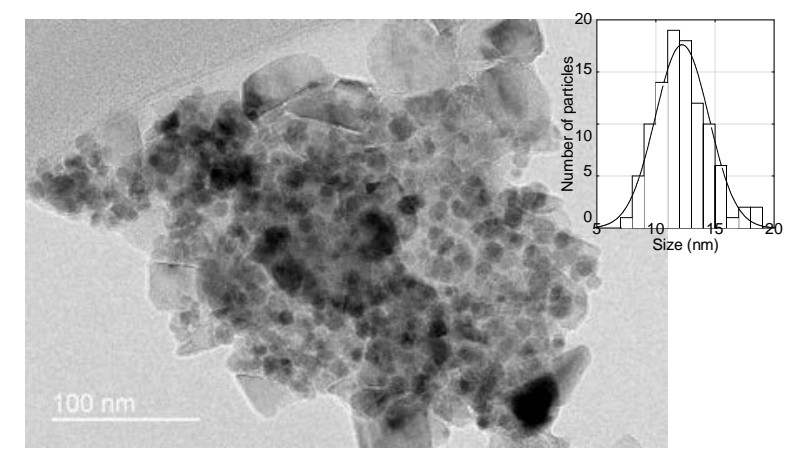

Figure 1

Fig. 1. TEM image of the diamond nano-crystals (large objects of irregular shapes) and the single-domain magnets (smaller and darker round objects) covering the surface of the diamonds. The inset shows approximately Gaussian (the Gaussian with a mean of $12 \mathrm{~nm}$ and standard deviation of $2 \mathrm{~nm}$ is shown as a solid line in the inset) size distribution of nano-magnets.

An example of an optical image is shown in Fig. 2 (left panel) where darker spots 
correspond to stronger luminescence. The image analysis shows that a very large majority of the diamonds is located on gold electrodes used for running RF current in the ODMR experiments. The gold electrode is seen as a band in the bottom part of the image (below approximately 33 microns on the vertical scale).
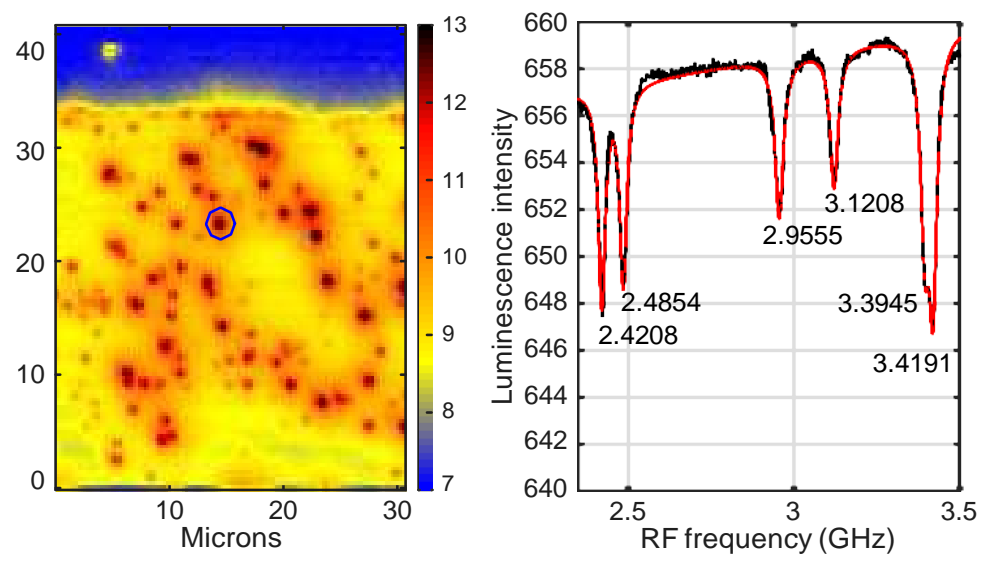

Figure 2

Fig. 2. Left panel shows wide-field optical image of the diamonds (dark-red diffraction limited spots) on a substrate. The colorbar on the right to the image represents a natural logarithm of the luminescence intensity. The right panel is an ODMR spectrum of $\mathrm{NV}^{-}$ centres in a selected diamond crystal indicated by a circle in the left panel. The external magnetic field is about $21 \mathrm{mT}$. The numbers show frequencies of the six RF transitions.

To investigate the magnetic properties of the sample, it has been placed in an external magnetic field. The wide-field imaging allows simultaneous detection of ODMR spectra of many diamonds (all diamonds in the field of view) under nearly identical conditions. The right panel in Fig. 2 shows ODMR spectrum of a single diamond enclosed in a circle in the left panel. The solid theoretical curve is a sum of six Lorenzians and a straight line. The straight line represents the base level of the luminescence intensity (the intensity which is not affected by the RF selective repopulation of the $m= \pm 1$ spin states). The six lines in the spectrum indicate the presence of the NV centres with three different orientations relative to the magnetic field because only three out of four theoretical possibilities are realised in this particular crystal. In other crystals we could identify 1 to 4 directions of NV axes.

In principle, each diamond crystal can poses NV-centers with four distinct but symmetry 
equivalent orientations of the $\mathrm{N}-\mathrm{V}$ axis relative to the crystal axes. But not all these possibilities are realised in each crystal. The formation of NV-centers is a quasi random process and some of the four directions may be represented by several centers while other possibilities may be not realised at all. Significant statistical fluctuations in the distribution of the small number centers (typically about 15 per crystal) between the four possibilities is expected. All the centers with the same orientation produce a pair of lines in an ideal crystal when ODMR spectra are taken in external magnetic fields. These lines are positioned symmetrically relative to the line at zero-field if the field is oriented strictly parallel to the center axes or if the field strength is small so that the linear approximation holds. ${ }^{31}$ In our experiments neither of the two conditions holds and the positions of the two lines are asymmetrical and their relative intensities are different.

The ODMR frequencies have been used to find the parameters of an emprical Hamilto$\operatorname{nian}^{22}$

$$
\hat{\mathrm{H}}=\underset{z}{D \hat{S}^{2}}+\underset{y}{E}\left(\hat{S}^{2}-\hat{S}^{2}\right)+\underbrace{\mu_{B}}_{\mathrm{h}} B \cdot \hat{S}
$$

where $\mu_{\mathrm{B}} / \mathrm{h} \approx 13.996 \mathrm{GHzT}^{-1}$ is the Bohr magneton, $\mathrm{g} \approx 2.0023$ is the electron $\mathrm{g}$-factor, $\hat{S}_{x, y, z}$ and $\hat{S}$ are the $S=1$ spin operators. The strength of the magnetic field $B$ and the angles between the magnetic field vector and the directions of the NV axes are the fitting parameters. The values of $\mathrm{D} \approx 2870 \mathrm{MHz}$, the zero-field fine-structure splitting (associated mainly with spin-spin interactions) and the crystal-strain related parameter $\mathrm{E} \leq 5 \mathrm{MHz}$ (the stress breaks the $C_{3 v}$ symmetry of the centre and splits $m= \pm 1$ levels) have been found from zero-field ODMR spectra which also have been measured for each crystals. The relative orientations of $\mathrm{NV}$ axes determined from the fitting are consistent with the orientation expected for orientations of the NV axes in a single diamond crystal. The consistency confirms that the white spots in the figure represent single crystal of diamond.

The strength of the local magnetic field has been measured with 15 crystals for four settings of the field. The strength of the field measured by a commercial magnetometer and confirmed by numerical modelling of the field with magnetisation of the permanent magnet 


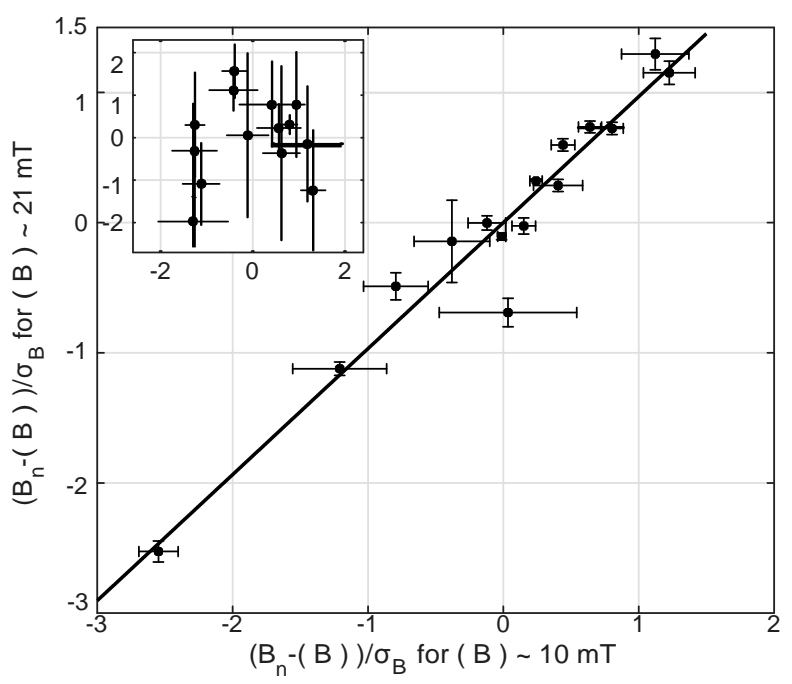

Figure 3

Fig. 3. Scatter plot for the field estimated by different crystals distributed within $30 \times 30$ microns image area. The plot shows deviation of the estimated values (15 sample data) from the sample mean divided by the sample standard deviation. The figure shows clear correlation between the high field values and low field values. The straight line is the linear fit with a gradient of $0.97 \pm 0.15$. The inset shows results obtained when no magnets are attached to the nano-diamonds. The scatter shows no statistically significant correlation.

being a single fitting parameter was $5.15 \pm 0.15,10.6 \pm 0.4 \mathrm{mT}, 21.3 \pm 0.9 \mathrm{mT}$ and $40.8 \pm 1.6$ $\mathrm{mT}$ (we will refer to these fields as $5 \mathrm{mT}, 10 \mathrm{mT}, 21 \mathrm{mT}$ and $40 \mathrm{mT}$ settings in the following discussion). A scatter correlation plot for two settings is shown in Fig.3. It is clear that there is a very strong correlation in the distribution of the data points. If a crystal detects a stronger field at $10 \mathrm{mT}$ setting, then the same crystal detects a stronger than average field at $20 \mathrm{mT}$ setting. Note that these crystals have been distributed within a small region of about $30 \times 30$ micros and the variation of the external magnetic field within this area is smaller than $0.01 \%$ according to our numerical simulations. Such correlation could result from a number of reasons intrinsic to the diamond crystals. For example, strong distortion of the crystal lattice in nm-sized crystals or surface proximity effects. But the inset in Fig.3 shows no statistically significant correlation if the diamond crystals are free from the nano-magnets. Therefore we conclude that the correlation results from the presence of the single-domain magnets. 
To explain the correlation we need to take into account that the single-domain magnets have a nonzero average magnetisation in the presence of an external magnetic field (otherwise the magnetisation spontaneously changes its direction and averages to zero). The averaged over time magnetic moment (for the case of the external field being aligned with the direction of easy magnetisation) reads ${ }^{1}$

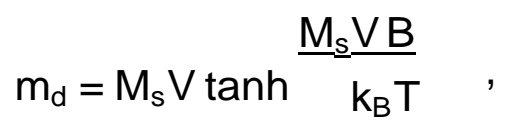

where $M_{s}$ is the instantaneous (or saturated) magnetisation of the domain, $V$ is its volume $\left(\mathrm{M}_{\mathrm{S}} \vee\right.$ being the instantaneous magnetic moment of the domain), $\mathrm{k}_{B}$ is the Boltzmann constant, and $T$ is the temperature. The magnetic moment is proportional to the external magnetic field if the field is much smaller than $B_{0} \equiv k_{B} T /\left(M_{S} V\right)$. While the value of $M_{S}$ depends on the size of the domain very little (but increases with the size), the dependence on the volume makes $\mathrm{B}_{0}$ quickly decreasing with the domain diameter. The contributions of the nano-magnets to the total field also varies from crystal to crystal because the number of magnets and their position on the surface as well as the shape of the crystals vary. This explains why the local fields measured with different crystal vary as seen in Fig.3. But the deviation of the local field from its mean value, $\Delta \mathrm{B}$ will be proportional to the strength of the external field despite of these variations. This proportionality explains the strong correlation between the values of $\Delta \mathrm{B}$ measured with the same crystal at different external fields.

At fields comparable to $B_{0}$ and higher, the magnetic moment saturates and does not increase as quickly as the external field strength. Therefore the relative contribution of the nano-magnets to the total magnetic field, $\Delta B / B \propto m_{d} / B$ decreases. Therefore it is expected that the value of $\Delta B /(B)$ will decrease for every diamond crystal as the field increases. The magnitude of this decrease will be not exactly the same for all crystals as the saturation effect depends on the size of the magnetic domains and possible coupling between the domains if 
they sit close to each other. The measurements of $\Delta B /(B)$ are presented in Fig. 4 for 5, 10, 21, and $40 \mathrm{mT}$ settings.

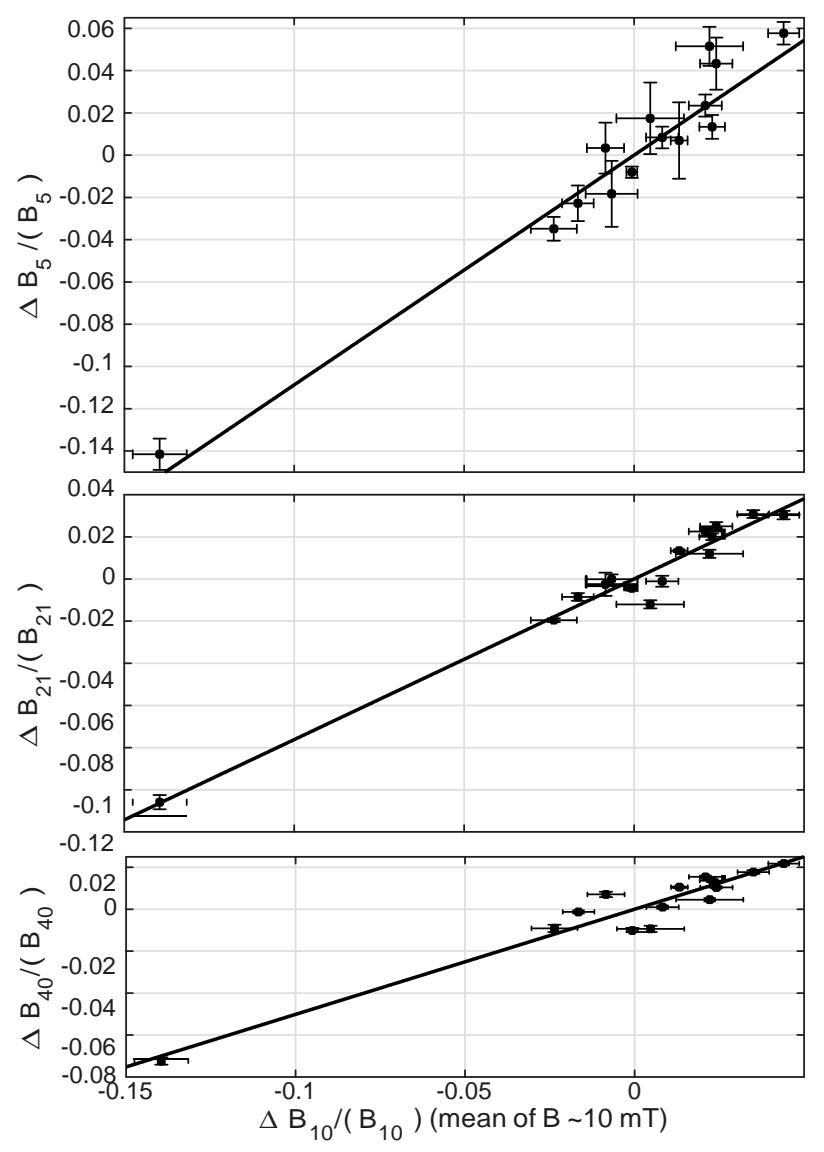

Figure 4

Fig. 4. Scatter plot of $\Delta B /(B)$, the relative deviations of magnetic field from (B) its mean value measured using different nano-diamonds distributed within about 30 microns wide area on the silica substrate. The sensitivity of the field measurements is indicated by the error bars which are on average $\pm 0.04 \mathrm{mT}$. The straight lines represent linear fits to the data points. The gradients of the lines are $1.09 \pm 0.17$ (top), $0.76 \pm 0.08$ (middle), and $0.50 \pm 0.09$ (bottom). The error bars are estimated from the noise in the ODMR spectra and the quality of the fit of the Lorentzian lines to the observed ODMR lines. The difference in the gradients between the middle panel and the other two is about three standard deviations (the corresponding confidence interval is 99.5\%). The change of the gradient is explained by the saturation of the single-domain magnetisation.

It is clear from the figure that the dispersion of the points in the vertical direction decreases as the field gets stronger (note, the vertical scale is the same in all panels). The theoretically expected gradient a can be estimated as follows 


$$
a=\frac{B_{h} \tanh \left(B_{v} / B_{0}\right)}{B_{v} \tanh \left(B_{h} / B_{0}\right)}
$$

where $B_{h}$ and $B_{v}$ are magnitudes of the two external fields (the results of the measurements with $B_{h}$ external field are used as the horizontal coordinates in the scatter plot and the values obtained with $B_{v}$ as the vertical coordinates). Given the average size of the magnets $(12 \mathrm{~nm})$ and a typical magnetisation of $3.1 \times 10^{5} \mathrm{~A} / \mathrm{m}\left(60 \mathrm{emu} / \mathrm{g}\right.$ or $\left.310 \mathrm{emu} / \mathrm{cm}^{3}\right),{ }^{32}$ the estimated value of $B_{0}$ is about $15 \mathrm{mT}$. This value is in a satisfactory agreement with the value obtained by measuring the magnetisation curve of the synthesised paramagnetic nanoparticles (see Supporting Information). The expected theoretical gradients estimated using Eq. (3) are then 1.1, 0.72, and 0.42 for the three panels (from the top and the bottom). These theoretical estimates are close to the experimental values of $1.09 \pm 0.17,0.76 \pm 0.08$ and $0.50 \pm 0.09$ obtained by fitting straight lines to the data points in the scatter plots as shown in Fig.4. Given that the size of the nano-magnets obtained from TEM images can be slightly different from the size estimated using measured average magnetisation the Langevin equation as observed by Li et al ${ }^{32}$ and that this size has about $20 \%$ variation in our sample, the agreement is good.

The direction of the field changes little because the contribution of a nano-magnet is not larger than $5 \%$ of the external field applied to the sample. The corresponding maximum change of the direction of the total field is less than 3 degrees (achieved if the field of a magnet is strictly perpendicular to the external field). But the field of many uniformly distributed nano-magnets is nearly parallel to the external field due to the approximate axial symmetry (around the external field direction) of the average magnetisation .

The ODMR spectra of the 15 crystals used in this study showed line broadening of about $10 \mathrm{MHz}$ at 40-mT external field. The broadening varied from crystal to crystal significantly. The broadening is observed because the field produced by nano-magnets has a different strength at the locations of different NV-centers (if there are several centers with an identical orientation within a single nanocrystal). A relatively small broadening has several qualitative 
explanations. First, most of the crystals in the field of view (see Fig. 2) show shallow and broad ODMR lines and could not be used for the purpose of the field measurements within the $5 \mathrm{mT}$ to $40 \mathrm{mT}$ range. The selected "good crystals" could be the crystals with approximately uniform field. Second, a permeable material uniformly covering a spherical shell creates inside the shell a uniform shielding field. ${ }^{33}$ Although we do not have such an ideal shielding, the variation of the field could be still small across each of the crystals. Third, the main contribution to the narrow ODMR lines comes from the centres located away from the surface, in the middle of the crystals where the field is most uniform.

As the last point of the discussion, we will estimate the field in the centre of the crystals. We can treat a single-domain magnet as a point dipole. Given an average radius of the crystals $r_{\mathrm{c}} \approx 20 \mathrm{~nm}$ and the radius of the nanomagnet $\mathrm{r}_{\mathrm{nm}} \approx 6 \mathrm{~nm}$, the approximate location of the centre of the crystal is at the distance of $r \approx r_{c}+r_{n m} \approx 26 \mathrm{~nm}$ from the magnet. The magnetic field of a nano-magnet then can be estimated as follows

$$
\mathrm{B}_{\mathrm{nm}} \approx \underset{4 \pi r^{3}}{\stackrel{\mu_{0}}{0}} \mathrm{M}_{\mathrm{s}} \mathrm{Vtanh} \quad \mathrm{B}_{0}^{\underline{B}}
$$

where $\mu_{0}$ is the vacuum permeability. Given $B_{0}=15 \mathrm{mT}$ and the external field of $10 \mathrm{mT}$, the contribution of a single domain is about $0.9 \mathrm{mT}$. By the order of magnitude, this value is in agreement with about $10 \%$ range of the relative deviations from the mean value observed at $10 \mathrm{mT}$ setting (see Fig.4).

\section{Conclusion}

In this paper we have presented synthesis of novel composite particles. These particles are made of chemically bound luminescent nano-diamonds and single-domain magnets. We have developed a method based on nano-magnetometry (using ODMR of negatively charged NV centers embedded in the bound to magnets diamond nano-crystals) which clearly demonstrates the presence of the magnets on the surface of the nano-diamonds. The ODMR also 
shows saturation of the average magnetisation of the nano-magnets in external fields from 10 to $40 \mathrm{mT}$, in agreement with a simple model of single-domain magnetisation. A relatively high homogeneity of the local fields created by the magnets has been explained qualitatively but needs further research to obtain a quantitative agreement. These results demonstrate a new class of composite nano-particles with potential applications in a variety of fields such as intracellular diagnostics, hypothermal therapy, and computing.

\section{Acknowledgement}

This work has been supported by ARC grants DP0771676, DP130102699, and DP130102274. We acknowledge Chunsheng Fang for helping with magnetisation measurements.

\section{Supporting Information Available}

Supporting Informations shows HRTEM, XRD, SAED, and the magnetisation data measured for the super-paramagnetic particles. The magnetisation curve is compared to the classical Langevin function. This material is available free of charge via the Internet at http: //pubs.acs.org/.

\section{References}

(1) Cullity, B. D.; Graham, C. D. Introduction to Magnetic Materials; John Wiley and Sons, Inc: Hoboken, New Jersey, 2009.

(2) Weller, D.; Moser, A. Thermal Effect Limits in Ultrahigh-Density Magnetic Recording. IEEE Trans. Magn. 1999, 35, 4423-4439.

(3) Hütten, A.; Sudfeld, D.; Ennen, I.; Reiss, G.; Hachmann, W.; Heinzmann, U.; Wojczykowski, K.; Jutzi, P.; Saikaly, W.; Thomas, G. New Magnetic Nanoparticles for Biotechnology. J. Biotech. 2004, 112, 47-63. 
(4) Ito, A.; Shinkai, M.; Honda, H.; Kobayashi, T. Medical Application of Functionalized Magnetic Nanoparticles. J. Biosci. Bioeng. 2005, 100, 1-11.

(5) Becherer, M.; Kiermaier, J.; Breitkreutz, S.; Eichwald, I.; Ziemys, G.; Csaba, G.; a, D. S.-L. Towards On-Chip Clocking of Perpendicular Nanomagnetic Logic. Sol. State Electr. 2014, 102, 46-51.

(6) Kodama, R. H. Magnetic Nanoparticles. J. Mag. Mag. Meter. 1999, 200, 359-372.

(7) Wernsdorfer, W. Classical and Quantum Magnetization Reversal Studied in NanometerSized Particles and Clusters. Adv. Chem. Phys . 2001, 118, 99-190.

(8) Gossuin, Y.; Gillis, P.; Hocq, A.; Vuong, Q. L.; Roch, A. Magnetic Resonance Relaxation Properties of Superparamagnetic Particles. Adv. Rev. 2009, 1, 299-310.

(9) Wernsdorfer, W.; Orozco, E. B.; Hasselbach, K.; Benoit, A.; Barbara, B.; Demoncy, N.; Loiseau, A.; Pascard, H.; Mailly, D. Experimental Evidence of the Neel-Brown Model of Magnetization Reversal. Phys. Rev. Lett. 1997, 78, 1791-1794.

(10) Chudnovskiy, A.; Ch.Huebner,; Baxevanis, B.; Pfannkuche, D. Spin Switching: From Quantum to Quasiclassical Approach. Phys. Status Solidi b 2014, 251, 1764-1776.

(11) Davies, G. Vibronic Spectra in Diamond. J. Phys. C: Solid State Phys 1974, 7, 37973809.

(12) Davies, G.; Nazare, M. H.; Hamer, M. F. The H-3 (2.463 EV) Vibronic Band in Diamond -Uniaxial Stress Effects and Breakdown of Mirror Symmetry. Proc. R. Soc. Lond. A. 1976, 351, 245-265.

(13) Maurer, P. C.; Kucsko, G.; Latta, C.; Jiang, L. Y.; Bennett, N. Y.; Pastawski, S. D.; Hunger, F.; Chisholm, D.; Markham, N.; Twitchen, M. et al. Room -Temperature Quantum Bit Memory Exceeding One Second. Science 2012, 336, 1283-1286. 
(14) Beveratos, A.; Brouri, R.; Gacoin, T.; Villing, A.; Poizat, J.-P.; Grangier, P. Single Photon Quantum Cryptography. Phys. Rev. Lett. 2002, 89, 187901.

(15) Su, L.-J.; Fang, C.-Y.; Chang, Y.-T.; Chen, K.-M.; Yu, Y.-C.; Hsu, J.-H.; Chang, H.C. Creation of High Density Ensembles of Nitrogen-Vacancy Centers in Nitrogen-Rich Type Ib Nanodiamonds. Nanotechnology 2013, 24, 315702.

(16) Rittweger, E.; Han, K. Y.; Irvine, S. E.; Eggeling, C.; Hell, S. W. STED Microscopy Reveals Crystal Colour Centres With Nanometric Resolution. Nature Photonics 2009, 3, 144-147.

(17) Dolde, F.; Fedder, H.; Doherty, M. W.; Nöbauer, T.; Rempp, F.; Balasubramanian, G.; Wolf, T.; Reinhard, F.; Hollenberg, L. C. L.; Jelezko, F. et al. Electric-Field Sensing Using Single Diamond Spins. Nature Physics 2011, 7, 459-463.

(18) Neumann, P.; Jakobi, I.; Dolde, F.; Burk, C.; Reuter, R.; Waldherr, G.; Honert, J.; Wolf, T.; Brunner, A.; Shim, J. H. et al. High-Precision Nanoscale Temperature Sensing Using Single Defects in Diamond. Nano Letters 2013, 13, 2738-2742.

(19) Kucsko, G.; Maurer, P. C.; Yao, N. Y.; Kubo, M.; Noh, H. J.; Lo, P. K.; Park, H.; Lukin, M. D. Nanometre-Scale Thermometry in a Living Cell. Nature 2013, 500, 5458.

(20) Plakhotnik, T.; Doherty, M. W.; Cole, J. H.; Chapman, R.; Manson, N. B. All-Optical Thermometry and Thermal Properties of the Optically Detected Spin Resonances of the NV-Center in Nanodiamond. Nano Letters 2014, 14, 4989-4996.

(21) Plakhotnik, T.; Aman, H.; Chang, H.-C. All-Optical Single-Nanoparticle Ratiometric Thermometry with a Noise Floor of $0.3 \mathrm{~K} \mathrm{~s}^{1 / 2}$. Nanotechnology 2015, 26, 245501.

(22) Doherty, M. W.; Manson, N. B.; Delaney, P.; Jelezko, F.; Wrachtrupe, J.; Hollen- 
berg, L. C. The Nitrogen-Vacancy Colour Centre in Diamond. Phys. Rep. 2013, 528, $1-45$.

(23) Taylor, J. M.; Cappellaro, P.; Childress, L.; Jiang, L.; Budker, D.; Hemmer, P. R.; Yacoby, A.; Walsworth, R.; Lukin, M. D. High-Sensitivity Diamond Magnetometer with Nanoscale Resolution. Nature Physics 2008, 4, 810-816.

(24) Maze, J. R.; Stanwix, P. L.; Hodges, J. S.; Hong, S.; Taylor, J. M.; Cappellaro, P.; Jiang, L.; Dutt, M. V. G.; Togan, E.; Zibrov, A. S. et al. Nanoscale Magnetic Sensing with an Individual Electronic Spin in Diamond. Nature 2008, 455, 644-647.

(25) Mamin, H. J.; Kim, M.; Sherwood, M. H.; Rettner, C. T.; Ohno, K.; Awschalom, D. D.; Rugar, D. Nanoscale Nuclear Magnetic Resonance with a Nitrogen-Vacancy Spin Sensor. Science 2013, 339, 557-560.

(26) Gould, M.; Barbour, R. J.; Thomas, N.; Arami, H.; Krishnan, K. M.; Fu, K.-M. C. Room-Temperature Detection of a Single $19 \mathrm{~nm}$ Super-Paramagnetic Nanoparticle with an Imaging Magnetometer. Appl. Phys. Lett. 2014, 105, 072406.

(27) Rondin, L.; Tetienne, J.-P.; Hingant, T.; Roch, J. F.; Maletinsky, P. Magnetometry with Nitrogen-Vacancy in Diamond. Rep. Prog. Phys. 2014, 77, 056503.

(28) Steinert, S.; Dolde, F.; Neumann, P.; Aird, A.; Naydenov, B.; Balasubramanian, G. High Sensitivity Magnetic Imaing Using an Array of Spins in Diamond. Rev. Sci. Instrum. 2010, 81, 043705.

(29) Kolen’ko, Y. V.; Bañobre-López, M.; Rodríguez-Abreu, C.; Carbó-Argibay, E.; Sailsman, A.; Piñeiro-Redondo, Y.; Cerqueira, M. F.; Petrovykh, D. Y.; Kovnir, K.; Lebedev, O. I. et al. Large-Scale Synthesis of Colloidal $\mathrm{Fe}_{3} \mathrm{O}_{4}$ Nanoparticles Exhibiting High Heating Efficiency in Magnetic Hyperthermia. J. Phys. Chem. C 2014, 118, 8691-8701. 
(30) Zhong, J.; Liu, W.; Kong, L.; Morais, P. C. A New Approach for Highly Accurate, Remote Temperature Probing Using Magnetic Nanoparticles. Sci. Reports 2014, 4, 6338.

(31) Maertz, B. J.; Wijnheijmer, A. P.; Fuchs, G. D.; Nowakowski, M. E.; Awschalom, D. D. Vector Magnetic Field Microscopy Using Nitrogen Vacancy Centers in Diamond. Appl. Phys. Lett. 2010, 96, 092504.

(32) Li, Z.; Tan, B.; Allix, M.; Cooper, A. I.; Rosseinsky, M. J. Direct Coprecipitation Route to Monodisperse Dual- Functionalized Magnetic Iron Oxide Nanocrystals Without Size Selection. Small 2008, 4, 231-239.

(33) Jackson, J. D. Classial Electrodynamics, 3rd ed.; John Wiley and Sons, Inc.: New York, 1998. 


\section{Graphical TOC Entry}

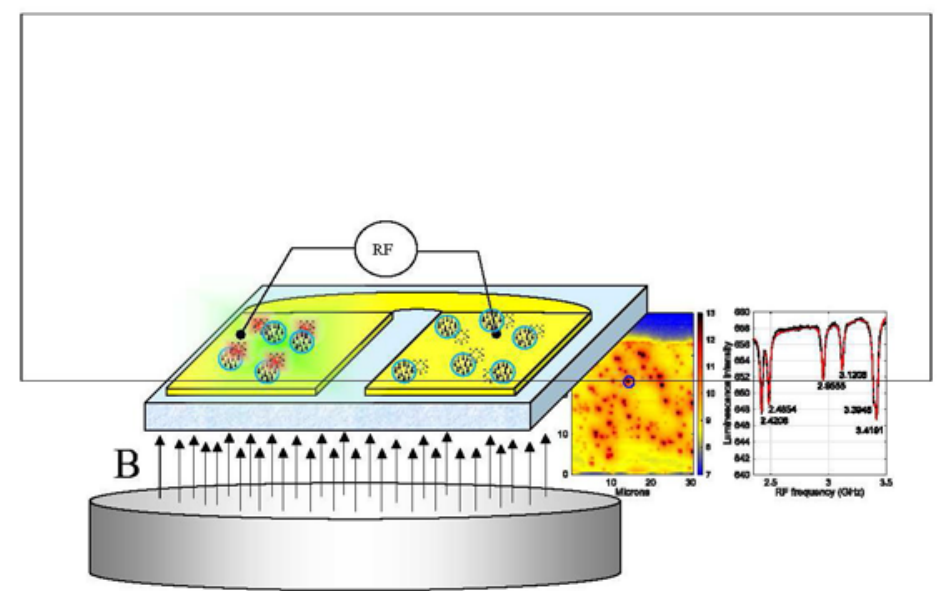

